\title{
HPA-Axis and Inflammatory Reactivity to Acute Stress is Related with Basal HPA-Axis Activity
}

Xuejie Chen ${ }^{1}$, Danielle Gianferante ${ }^{1}$, Luke Hanlin ${ }^{1}$, Alexander Fiksdal ${ }^{1}$, Juliana G. Breines ${ }^{1}$, Myriam V. Thoma ${ }^{1,2}$, Nicolas Rohleder* 1,3

${ }^{1}$ Department of Psychology and Volen National Center for Complex Systems, Brandeis University, Waltham, MA, United States

${ }^{2}$ Division of Psychopathology and Clinical Intervention, University of Zurich, Zurich, Switzerland

${ }^{3}$ Chair of Health Psychology, Friedrich-Alexander-University Erlangen-Nuremberg, Erlangen, Germany

Running Head: Basal HPA Axis Activity and Stress Reactivity

To whom correspondence should be addressed:

Prof. Dr. Nicolas Rohleder

Chair of Health Psychology

Friedrich-Alexander-University Erlangen-Nürnberg (FAU)

Nägelsbachstr. 49a

91052 Erlangen

Germany

Phone: +49 91338528990

Email: nicolas.rohleder@fau.de

(C) 2017. This manuscript version is made available under the Elsevier user license http://www.elsevier.com/open-access/userlicense/1.0/ 


\section{Abstract}

Introduction: Inflammation is drawing attention as pathway between psychosocial stress and health, and basal HPA axis activity has been suggested to exert a consistent regulatory influence on peripheral inflammation. Here we studied the relationship between basal HPA axis activity and inflammatory and HPA axis acute stress reactivity.

Methods: We recruited 48 healthy individuals and collected saliva for diurnal cortisol sampling at 6 points. Participants were previously exposed to the Trier Social Stress Test (TSST) on two consecutive days. Plasma interleukin-6 (IL-6) and salivary cortisol reactivity to acute stress were measured, and their relationships with basal activity were analyzed.

Results: Steeper cortisol awakening response (CAR) linear increase was related with stronger cortisol stress reactivity $(\gamma=0.015 ; p=0.042)$ and marginally significantly with greater habituation $(y=0.01 ; p=0.066)$. Greater curvilinearity of CAR was related with stronger cortisol reactivity $(\gamma=-0.014 ; p=0.021)$ and greater cortisol habituation $(\gamma=-0.011 ; p=0.006)$. Steeper daily linear decline was related with significant or marginally significantly stronger cortisol and IL-6 reactivity (cortisol: $\gamma=-0.0004 ; p=0.06$; IL-6: $\gamma=-0.028 ; p=0.031$ ) and greater habituation (cortisol: $\mathrm{V}=-0.002 ; p=0.009, \mathrm{IL}-6: \mathrm{V}=-0.015 ; \mathrm{p}=0.033)$. Greater curvilinearity of daily decline was related with stronger IL-6 reactivity $(\mathrm{y}=0.002 ; \mathrm{p}=0.024)$ and also greater cortisol and IL-6 habituation (cortisol: $y=0.00009 ; p=0.03, I L-6: y=0.001 ; p=0.024$ ).

Conclusions: Patterns of basal HPA axis activity that are related with healthier outcomes were found to be related with stronger initial cortisol and IL-6 reactivity and greater habituation. This is an important step in understanding the long-term health implications of acute stress responsiveness, and future studies should employ longitudinal designs to identify the direction of these relationships. 


\section{Introduction}

Psychological stress is related to the onset of a spectrum of diseases (Cohen et al., 2007). In human beings, acute stress activates the sympathetic nervous system (SNS) and hypothalamus pituitary adrenal (HPA) axis. The SNS is activated almost immediately after stress and increases the involuntary processes required to handle challenge, while HPA axis is activated in minutes and has additional effects that may compensate, enhance, or suppress the SNS effects (Chrousos and Gold, 1992; Sapolsky et al., 2000).

\subsection{Stress Response and Health}

There are marked inter-individual differences in stress reactivity of these two systems. In a seminal article, McEwen (1998) suggested several maladaptive types of stress reactivity that contribute to allostatic load. These include inadequate response to stress, failure to shut off the HPA axis and SNS after stress, and lack of habituation to repeated stressors (McEwen, 1998). These interpersonal differences in stress reactivity are relatively stable and their health implications were investigated by several studies (Cohen and Hamrick, 2003). Alterations in HPA axis stress reactivity were found to be related with certain diseases, including atopic dermatitis (Buske-Kirschbaum et al., 2002), depression (Burke et al., 2005), diabetes mellitus (Siddiqui et al., 2015), incident hypertension (Hamer and Steptoe, 2012), and childhood cancer (Laufer et al., 2012). Individual differences in habituation to stress are also important in terms of health, with participants showing lesser HPA axis habituation reporting lower self-esteem, more physical symptoms (Kirschbaum et al., 1995), and greater vital exhaustion (Kudielka et al., 2006).

Recently there has been increasing interest in exploring inflammation as a potential pathway linking stress and health, especially considering that inflammation is reliably induced by stress and directly affects various organ systems (Rohleder, 2014). Previous studies showed that peripheral inflammation contributes to increased risk of several diseases, including 
myocardial infarction (Ridker et al., 2000), diabetes (Wellen and Hotamisligil, 2005), and depression (Dantzer et al., 2008). In one longitudinal study, over-exaggerated acute inflammatory stress response was prospectively associated with increased blood pressure three years later (Brydon and Steptoe, 2005).

\subsection{Basal HPA Axis Activity and Health}

In addition to reactivity to acute stress, the HPA axis is also characterized by a pronounced circadian rhythm of basal activity (Weitzman et al., 1971). Diurnal cortisol activity is considered to be subjected to the influence of allostatic load and has been recognized as an allostatic load algorithmic formulation (Juster et al., 2010; McEwen and Stellar, 1993). Basal activity is usually broken down into two components: A rapid increase during the first hour after waking referred to as cortisol awakening response (CAR), and a gradual decrease throughout the day called the daily decline (Kirschbaum and Hellhammer, 1989; Pruessner et al., 1997b; Wust et al., 2000). CAR and daily decline are related with relatively independent processes (Edwards et al., 2001; Schmidt-Reinwald et al., 1999). CAR is conceptualized as a response to awakening (Pruessner et al., 1997a; Schmidt-Reinwald et al., 1999; Wetherell et al., 2015a). Operationalized as cortisol output measured by Area Under Curve relative to ground (AUCg) and Area Under Curve relative to increase (AUCi) of CAR was found to be related with health status, with lower CAR being associated with unfavorable disease courses of anxiety and depressive disorders in a two-year longitudinal study (Vreeburg et al., 2013), and with chronic stress in healthy male participants (Duan et al., 2013). It is noteworthy to point out that sometimes this relationship is more complicated and can be non-linear. For example, individuals with low or mild levels of depression showed a normal AUC of CAR, while individuals with moderate level of depression showed increase CAR AUC, and individuals with severe level of CAR showed decreased CAR AUC (Veen et al., 2011). In general, higher CAR seems to be related with exposure to psychological stressors, and lower CAR with adverse health consequences (Chida and Steptoe, 2009). Cortisol daily decline, on the other hand, can be 
conceptualized as an indication of negative feedback loop of HPA axis and the ability to recover from daily stress at the end of day (Dmitrieva et al., 2013).

While AUC depicts the total output of cortisol, the profile of both, CAR and daily decline, can also be described by three components: a beginning value, a slope depicting the rate of linear increase or decrease, and a quadratic term together term describing curvilinearity - that is, the tendency to slow down or even reverse both the CAR linear increase rate and cortisol daily decline rate (Adam, 2006; Adam et al., 2006; Slatcher et al., 2015). Typically, a steeper linear daily decline was related with better health conditions. In longitudinal studies, flatter daily decline predicted adverse health outcomes like early mortality in cancer patients (Sephton et al., 2013; Sephton et al., 2000). It was also found to predict survival in epithelial ovarian cancer (Schrepf et al., 2015), all-cause mortality (Kumari et al., 2011), and incidence of type 2 diabetes (Hackett et al., 2016). Cross-sectionally, flatter cortisol was related with more depression symptom in midlife women (Knight et al., 2010). At the molecular level, flatter daily curves were related with cellular aging measured by telomerase (Tomiyama et al., 2012), and traumatic experience-induced DNA methylation of the human glucocorticoid receptor (Labonte et al., 2014). Flatter daily slope was also reliably found associated with chronic stress (Miller et al., 2007). Greater curvilinearity of daily decline was related with more favorable psychological characteristics such as lower loneliness and higher executive function (Doane and Adam, 2010; Stawski et al., 2011). For CAR, Cortisol levels at 30 minutes post-awakening were significantly lower in healthy young male adults with higher perceived stress and anxiety (Duan et al., 2013), and cortisol levels either at awakening or 30 to 40 minutes post-awakening were negatively related with slope of daily decline (Adam and Kumari, 2009).

\subsection{Associations between basal HPA axis activity with stress reactivity}

Based on the evidence summarized above, we propose a model in which individual differences in acute stress reactivity and habituation are related with basal HPA axis activity, 
and have health implications mediated through consistent effects on basal activity. Surprisingly, only a few studies have addressed their interrelationship directly and results were not consistent. For example, several studies found no relationship between CAR and cortisol responses to the Trier Social Stress Test (TSST) (Kidd et al., 2014; Schmidt-Reinwald et al., 1999), but another study found a negative relationship between CAR and cortisol response to uncontrollable noise in adult women (Quirin et al., 2008). Regarding daily cortisol, one study reported a positive correlation between cortisol reactivity and AUC of daily cortisol but no correlation with the daily slope (Kidd et al., 2014). To the best of our knowledge, no study has tested the relationship between HPA axis habituation to repeated stress and basal HPA axis activity, and no research has addressed the relationship between peripheral inflammatory reactivity and basal HPA axis activity.

\subsection{Research aims and hypotheses}

The goal of the present research therefore was to examine the relationship between stress response patterns during first and repeated exposure and basal HPA axis activity. We hypothesized that individuals with greater habituation of cortisol and IL-6 to repeated stress would show steeper linear slopes of CAR and daily decline, as well as greater curvilinearity. We did not have directional hypotheses about the relationship between basal cortisol patterns and initial cortisol and IL-6 stress reactivity.

\section{Methods}

\subsection{Sample}

Data for this article were from a larger research project studying the effects of stress on aging, which had been carried out in the Laboratory for Biological Health Psychology at Brandeis University for over two years (Breines et al., 2014; Gianferante et al., 2014). Participants ranged in age from 18 to 65 years and were recruited from the Brandeis University 
student population and the Greater Boston area. Interested volunteers were screened to be (1) within normal BMI range of $18-35 \mathrm{~kg} / \mathrm{m}^{2}$; (2) non-smokers; (3) free of any cardiovascular or other chronic diseases, as well as any medication that would interfere with measurement of stress hormones and inflammatory molecules; (4) free of any psychiatric disease, and did not report taking any psychiatric medication; (5) no previous exposure to the stress protocol; (6) female participants needed to be free of hormonal contraceptives, to report normal and regular menstrual cycles, and to be in luteal phase of menstrual when the laboratory stress took place.

In this report, we included data from 48 participants who completed both days of acute stress testing and at-home saliva collection. Thirty-three of these were in a younger age group (18 male, 15 female, age $21.30+-4.43$ years, BMI $24.27+-3.02 \mathrm{~kg} / \mathrm{m}^{2}$ ), and 15 in an older age group (6 male, 9 female, age $54.47+-4.42$ years, BMI $25.08+-3.33 \mathrm{~kg} / \mathrm{m}^{2}$ ). Of all participants, 25.0\% were Asian or Asian American, 4.2\% were Black or African American, $56.3 \%$ were White or Caucasian American, $4.2 \%$ were more than one race, and $10.3 \%$ did not specify race. Further details are shown in Table 1. The study protocol was approved by the Institutional Review Board and participants received monetary compensation.

\subsection{Procedure}

Eligible participants were scheduled for two successive laboratory sessions. All laboratory sessions were scheduled in the afternoon to control for circadian variation of stress hormones. In both laboratory sessions, a venous catheter was placed into an antecubital vein of the non-dominant arm, followed by a resting period of 30 minutes. First blood and saliva samples were taken and participants were exposed to a standard laboratory stress procedure. Further blood and saliva samples were taken immediately, as well as 10, 30 (saliva only), 60, and 120 minutes after stress. Home cortisol sampling was taken on two consecutive days in a two-week period following the laboratory stress. The home cortisol sampling procedure asked 
the participant to collect saliva within a week after the laboratory part of the study, during 6 time points on two successive days: immediately after awakening, 0.5, 1, 4, 9 and 13 hours after awakening. We asked participants to record the actual time when saliva samples were collected using a paper-and-pencil diary, and participants demonstrated good compliance. Data of participants who collected the awakening sample with a delay more than 5 minutes were excluded.

\subsection{Stress Induction Paradigm}

The Trier Social Stress Test (TSST) was performed in front of two panel members and a video camera; it consists of an anticipation period, public speaking, and mental arithmetic task in front of two panel members and a video camera each for 5 minutes. This procedure reliably activates biological stress systems and has been established as a standard procedure for inducing stress in adult participants (Kirschbaum et al., 1993; Kudielka et al., 2007). The themes of the public speech were the same on both days. To avoid effects of learning, the initial number and steps for the serial subtraction tasks were minimally modified on day 2 as previously described (Schommer et al., 2003).

\subsection{Assessment of HPA Axis (Re)activity}

As an indicator for HPA axis activation, we measured free cortisol in saliva. Saliva was collected by participants using Salivette collection devices (Sarstedt, Newton, NC, USA). Salivettes were stored at $-20^{\circ} \mathrm{C}$ until completion of the study, thawed, and centrifuged at 2,000 $\mathrm{g}$ for $5 \mathrm{~min}$. The resulting saliva was analyzed using a commercial chemiluminescence immuno assay (CLIA; IBL-Hamburg, Hamburg, Germany) with a lower detection limit of $0.41 \mathrm{nmol} / \mathrm{l}$. Intra- and inter-assay coefficients were below $10 \%$. 


\subsection{Assessment of Peripheral Inflammation}

Plasma IL-6 was measured as an indicator of peripheral inflammation. Blood samples were transported to the laboratory immediately after blood draw and centrifuged for 10 minutes at $1800 \mathrm{~g}$ and $4^{\circ} \mathrm{C}$. Plasma was divided into aliquots and stored at $-80^{\circ} \mathrm{C}$ until further analysis. IL-6 concentrations were quantified in duplicates using commercial high-sensitivity ELISA kits (Quantikine HS human IL-6; R\&D Systems; Minneapolis, MN, USA) with a minimum detectable concentration of $0.039 \mathrm{pg} / \mathrm{ml}$. Inter- and intra-assay variability was below $10 \%$.

\subsection{Statistical Analysis}

For diurnal cortisol activity, the values of each time point across the two days were averaged to obtain a basal diurnal curve. Repeated measures ANOVA with the within-subjects factor time was used to test for diurnal changes in basal HPA axis activity. To test for stressinduced increases of cortisol and IL-6, and habituation of these responses, we used repeated measures ANOVAs with the within-subject factors day and time. Greenhouse-Geisser correction was used if the assumption of sphericity was violated. Cortisol responses to each of the two stress tests were computed as the difference between peak value cortisol (highest cortisol level of the three samples taken 10, 30 and 60 minutes post stress) and baseline cortisol (right before TSST). IL-6 responses to each of the two stress tests were computed by subtracting IL-6 concentration at 120 minutes after stress from baseline. Time points for computation of these change variables were chosen based on previous research showed that cortisol after stress peaks 20 to 40 minutes post- stress (Dickerson and Kemeny, 2004) and plasma IL-6 peaks at approximately $120 \mathrm{~min}$ after stress (von Kanel et al., 2006b). Our findings were consistent with this pattern. Habituation of cortisol and IL-6 was defined as response to first exposure minus response to second exposure. 
To determine the factors that would affect diurnal cortisol curve, we used the hierarchical linear modeling approach (HLM; (Bryk and Raudenbush, 1992). HLM was chosen here because of the nested characteristics of our data: each participant had a set of daily cortisol values. We used both linear terms and quadratic terms for time to depict slope and curvilinearity of daily profile. Due to the skewed characteristics of diurnal cortisol values, log-transformation was applied.

The first level represented the 6 repeated, averaged cortisol measures within each day (within-person). In the following formulas, HCORTLG represents log-transformed salivary cortisol measured at home, and TIME_SQ stands for measurement time (since waking) squared.

$$
H C O R T L G_{i j(2.3 .9)}=\beta_{0 j}+\beta_{1 j}^{*}\left(T I M E_{i j}\right)+\beta_{2 j}^{*}\left(T I M E \_S Q_{i j}\right)+r_{i j}
$$

The second level was the between-person level, and at that level demographic and health factors such as age, gender and BMI, and physiological factors such as cortisol reactivity, IL-6 reactivity, cortisol habituation, and IL-6 habituation were considered.

$$
\begin{aligned}
& \beta_{0 j}=Y_{00}{ }^{*} Y_{01}{ }^{*}(F A C T O R 1)+Y_{02}{ }^{*}(F A C T O R 2)+\ldots \ldots . Y_{0 j}{ }^{*}(F A C T O R j)+u_{0 j} \\
& \beta_{1 j}=\gamma_{10}+\mathrm{\gamma}_{11}{ }^{*}(\text { FACTOR } 1)+\mathrm{\gamma}_{12}{ }^{*}(F A C T O R 2)+\ldots \ldots . \mathrm{\gamma}_{1 j}{ }^{*}(F A C T O R j) \\
& \beta_{2 j}=\gamma_{20}+\gamma_{21}{ }^{*}(F A C T O R 1)+\gamma_{22}{ }^{*}(F A C T O R 2)+\ldots \ldots . \gamma_{2 j}{ }^{*}(F A C T O R j)
\end{aligned}
$$

Specifically, we built two separate two-level multilevel models for cortisol awakening response $(0,0.5 \mathrm{~h}$, and $1 \mathrm{~h}$ post-awakening $)$, and for daily decline $(0,+4,+9$, and $+13 \mathrm{~h}$ postawakening), respectively. Since we used higher level growth curve, we grand mean centered time. Each model was built by first establishing a null model, and then adding demographic and health predictors such as age group, gender, and BMI and finally adding physiological predictors such as cortisol and IL-6 reactivity, as well as their habituation. In each step we first 
added predictors simultaneously and then deleted non-significant ones. Due to the highly correlated characteristics of reactivity and habituation of both cortisol and IL-6 we tested them individually, instead of simultaneously, in order to avoid multi-collinearity issues. Model fit parameters like -2 log likelihood were also taken into consideration to determine the final model. This analysis gave us an estimation of all three components of CAR and daily decline. For CAR, the intercept term $\left(\beta_{0 j}\right)$ demonstrated the average value of cortisol 0.5 hours after awakening (since we centered time on 0.5 hour), the linear term $\left(\beta_{1 j}\right)$ depicted the slope of cortisol increase, and linear together with the quadratic term $\left(\beta_{2 j}\right)$ depicted the curvilinearity of time effect on CAR. For daily decline, the intercept term $\left(\beta_{0 j}\right)$ demonstrated the average value 6.5 hours after awakening (since we centered time on 6.5 hour), the linear term $\left(\beta_{1 j}\right)$ depicted the slope of cortisol decrease, and linear together with the quadratic term $\left(\beta_{0 j}\right)$ depicted the curvilinearity of time effect on daily decline. Analysis used restricted maximum likelihood estimation, while model fit comparison used full maximum likelihood estimation.

\section{Results}

\subsection{Cortisol and IL-6 Response to Initial and Repeated Acute Stress Exposure}

On both TSST days, cortisol and IL-6 increased significantly in response to stress (time effect: cortisol: $F_{1.726,86.322}=20.51, p<0.001 ;$ partial $\eta^{2}=0.291 ;$ IL-6: $F_{1.065,53.267}=47.39, p<$ 0.001; partial $\eta^{2}=0.487$ ). Cortisol response to repeated exposure was significantly lower than the initial response, indicating habituation (day ${ }^{*}$ time effect: $F_{2.128,112.774}=5.17, p=0.006$, partial $\left.\eta^{2}=0.09\right)$ while IL-6 showed a stress response with significantly higher overall IL-6 concentrations, indicating sensitization (day effect: $F_{1,59.87}=7.06, p=0.01$; partial $\eta^{2}=0.19$, day ${ }^{\star}$ time effect: $F_{1.16,61.40}=1.55, p=0.22$, partial $\left.\eta^{2}=0.03\right)$. (Figure 1A and 1B). Cortisol and IL6 habituation correlated significantly with their reactivity on both days, respectively. We also tested the effect of age group, gender, and BMI on cortisol and IL-6 increase of each day and habituation. Young participants had a higher cortisol increase after initial stress $\left(t_{49}=2.156\right.$, 
$\mathrm{p}=0.037$ ). BMI was a significant predictor of a more pronounced IL-6 increase after TSST 2 $(\beta=0.183, p=0.003)$ and less habituation $(\beta=0.149, p=0.050)$ even after controlling for age and gender.

\subsection{Basal HPA Axis Activity}

Results showed that cortisol concentrations changed significantly during the day, with increases during the first hour after awakening, and decreases throughout the rest of the day (time effect: $F_{2.39,126.56}=73.32, p<0.001$, partial $\eta^{2}=0.58$; Figure $1 C$ ).

\subsection{Predictors of Cortisol Awakening Response (CAR)}

The intra-class correlation (ICC) of the model without adding level-2 predictors including age-group, gender, BMI, cortisol and IL-6 initial response and habituation showed that $50 \%$ of the variance of CAR was within-person, confirming that HLM was an adequate approach to test for determinants of CAR.

\subsubsection{Demographic and Health Characteristics as Predictors of CAR}

CAR differed across age group and gender (Figure 2A). For young and old participants (solid vs. dotted line), the difference lay mainly in the intercept: young participants had higher levels of cortisol than older participants $\left(\gamma_{01}=0.15 ; \mathrm{SE}=0.05 ; \mathrm{p}=0.008\right)$, and this difference was consistent throughout the first hour. The difference between male and female participants lay in the linear terms of time $\left(Y_{01}=0.18 ; S E=0.07 ; p=0.011\right)$. The slope of cortisol increase in females was steeper than in males in the first hour. BMI was not a significant predictor of CAR, whether considered individually or together with age group and gender. 


\subsubsection{Cortisol Reactivity and Habituation as Predictors of CAR}

Independent of the effect of age and gender, cortisol response to the first stress exposure was related to both the CAR linear term and the quadratic term. People with higher cortisol responses to initial stress showed a steeper slope of CAR increase $\left(\gamma_{12}=0.015\right.$; $S E=$ $0.007 ; p=0.042)$. They also showed greater curvilinearity $\left(\gamma_{12}=-0.014 ; S E=0.006 ; p=0.021\right)$

(Figure 2B). Cortisol habituation was also related to the CAR linear and quadratic terms. Participants showing greater habituation showed steeper slope with marginal significance $\left(\gamma_{12}=\right.$ $0.01 ; \mathrm{SE}=0.005 ; \mathrm{P}=0.066)$, and greater curvilinearity $\left(\mathrm{\gamma}_{12}=-0.011 ; \mathrm{SE}=0.004 ; \mathrm{p}=0.006\right)$ (Figure 2C).

\subsubsection{IL-6 Reactivity and Habituation as Predictors of CAR}

Neither initial stress-induced ${ }_{(2.1 .2)}$ IL-6 increase, nor stress-induced IL-6 habituation was a significant predictor of CAR. All p's were $>0.05$, and the lowest p-value was IL-6 habituation as the predictor of intercept of CAR $\left(\gamma_{11}=-0.025 ; \mathrm{SE}=0.017 ; p=0.13\right)$.

\subsection{Predictors of Cortisol Daily Decline}

In the model without level-2 predictors including age-group, gender, BMI, cortisol and IL6 initial response and habituation, the intra-class correlation (ICC) showed that $12.5 \%$ of the total variance of cortisol daily decline was within-person, confirming that HLM was an adequate approach to test for determinants of daily decline.

\subsubsection{Demographic and Health Characteristics as Predictors of Cortisol Daily Decline}

Gender was a significant predictor of cortisol daily decline. Male participants showed a steeper linear cortisol decrease than female participants $\left(\gamma_{11}=0.013\right.$; SE $\left.=0.005 ; p=0.018\right)$. Age group was not a significant predictor and therefore was not included in the final model. BMI 
was not a significant predictor of cortisol daily decline, whether considered individually or together with age group and gender.

\subsubsection{Cortisol Reactivity and Habituation as Predictors of Cortisol Daily Decline}

Independent of the effect of gender, cortisol increase following the first stress exposure was related to the linear term of time. Participants with higher cortisol responses to initial stress showed a steeper daily declines which was marginally significant $\left(\gamma_{12}=-0.0004 ; S E=0.0002 ; p\right.$ $=0.06)$ (Figure $3 \mathrm{~A})$. Cortisol habituation was related to both the linear and quadratic terms of time. Participants who showed stronger habituation during the second stress exposure showed steeper daily declines $\left(\mathrm{Y}_{12}=-0.002 ; \mathrm{SE}=0.0006 ; \mathrm{p}=0.009\right)$, and greater curvilinearity $\left(\mathrm{V}_{12}=\right.$ $0.00009 ; \mathrm{SE}=0.00004 ; \mathrm{p}=0.030)$ (Figure 3B).

\subsubsection{IL-6 Reactivity and Habituation as Predictors of Cortisol Daily Decline}

Independent of the effect of gender, IL-6 increase following the first stress exposure was related to both linear and quadratic terms of time. Participants with higher IL-6 response to the first stress exposure showed steeper daily declines $\left(\gamma_{12}=-0.028 ; S E=0.013 ; p=0.031\right)$, and greater curvilinearity $\left(\mathrm{V}_{21}=0.002 ; \mathrm{SE}=0.0009 ; \mathrm{p}=0.024\right)$ (Figure 4A). IL-6 habituation during the second stress exposure also was related to both linear and quadratic term of time. Participants who showed stronger habituation during second exposure showed steeper linear daily declines $\left(\gamma_{12}=-0.015 ; \mathrm{SE}=0.007 ; \mathrm{p}=0.033\right)$, and greater curvilinearity $\left(\mathrm{\gamma}_{21}=0.001 ; \mathrm{SE}=\right.$ $0.0004 ; p=0.024)$ (Figure 4B).

\section{Discussion}

In the current study, we set out to test whether HPA axis and inflammatory reactivity to acute repeated stress was related with basal diurnal HPA axis activity. As expected, both the HPA axis and the inflammatory system were activated in response to acute stress. Upon 
repeated stress exposure, the HPA axis showed habituation but the inflammatory system did not, which is consistent with previous literature (Kemeny, 2003; Schommer et al., 2003; von Kanel et al., 2006a; von Kanel et al., 2006b). Basal HPA axis activity showed the same pattern as described in the literature, displaying a rapid increase within the first hour after waking and a gradual decline throughout the day (Kirschbaum and Hellhammer, 1989; Pruessner et al., 1997a).

With regard to relationships between basal HPA axis activity, acute cortisol stress responses, and cortisol habituation across repeated stressors, we found that a steeper CAR was associated with pronounced cortisol responses and greater cortisol habituation. This finding is consistent with our hypothesis that a more pronounced awakening response was related with adaptive acute reactivity. Furthermore, greater curvilinearity of CAR was associated with pronounced initial response and greater habituation of cortisol responses to repeated stress.

Participants with more pronounced initial cortisol response, stronger habituation of both cortisol and IL-6 showed steeper linear decline of diurnal cortisol. Again, this is consistent with our hypotheses, and could imply that a steeper linear daily decline is a more adaptive acute response pattern, consistent with previous studies demonstrating flatter daily declines predicting adverse health outcomes (Sephton et al., 2013). Participants with pronounced initial cortisol response and stronger habituation of both cortisol and IL-6 also showed a greater curvilinearity, which is consistent with previous findings that adverse psychological states were associated with less curvilinearity (Doane and Adam, 2010), while adaptive traits were related with greater curvilinearity (Stawski et al., 2011). Our finding of a positive relationship between steeper slope and more curvilinearity of daily decline and higher initial IL-6 reactivity was unexpected, and was in conflict with the general assumption that high inflammatory responses to acute stress are maladaptive and linked with disease risk (Brydon and Steptoe, 2005). However, the positive relationship found here might also point to a different function of inflammatory stress response: 
it could be considered adaptive, allowing for necessary preparation of host defense mechanisms, and only turn maladaptive if later down-regulation is inhibited.

\subsection{Potential Mechanisms}

Our findings show that response and habituation of HPA axis and inflammation were related with basal HPA axis activity. This can occur through several mechanisms. It has been proposed that both HPA axis and inflammatory systems, when activated repeatedly, can have negative long-term effects. In animal models it was observed that excess cortisol output had adverse effects on neural systems, especially hippocampal glucocorticoid receptors, leading to reduced feedback sensitivity of the HPA axis (Sapolsky, 1999). It has also been documented that the HPA axis was regulated by inflammation's feed forward effect: peripheral inflammatory factors can stimulate the HPA axis to increase cortisol production to exert an anti-inflammatory effect (Sternberg et al., 1992). Therefore, prolonged inflammation might promote chronic HPA axis activation at the level of the hypothalamus (Besedovsky et al., 1991; Dunn, 2000; Turnbull and Rivier, 1995). Furthermore, chronic stress was also related with changes in peripheral glucocorticoid sensitivity (Rohleder, 2012). These direct effects on the central nervous system and HPA axis circuits, including the amygdala, hippocampus and peripheral tissue glucocorticoid receptors, can be related with altered basal HPA axis activity. Stress reactivity patterns can also influence basal HPA axis activity indirectly through the multi-directional influence between HPA axis, immune system and circadian clock rhythm (Cermakian et al., 2014; Nader et al., 2010; Scheiermann et al., 2013).

The increase rate of CAR has been proposed to be a good measurement of responsiveness of the HPA axis (Pruessner et al., 1997a; Schmidt-Reinwald et al., 1999) and greater curvilinearity of CAR can facilitate the recovery of cortisol and be a manifestation of good recovery ability of the HPA axis. Our findings that stronger initial reactivity and more habituation of cortisol were associated with steeper slope and more curvilinearity of CAR 
therefore can be interpreted as a sign of better ability of the HPA axis to respond and to recover, not only at an acute reactivity level but also at a long-term basal level. Daily cortisol decline, on the other hand, can be conceptualized as an demonstration of negative feedback in HPA axis (Lightman and Conway-Campbell, 2010; Sarabdjitsingh et al., 2010) and reduced glucocorticoid sensitivity was found to be associated with flatter diurnal declines in females with major depressive disorder (Jarcho et al., 2013). Our study took both linear and nonlinear parts of the daily decline into consideration and showed that slower linear decrease and less curvilinearity of daily cortisol levels were related with acute response patterns suspected to be maladaptive, suggesting that even in healthy participants, people with relatively unhealthy acute stress response patterns may have daily declines characteristic of compromised negative feedback.

\subsection{Implications}

It has been demonstrated that stress reactivity patterns can have implications for health, but the mechanisms underlying these long-term effects are unknown. Compared to the acute stress response, basal activity exerts a more consistent effect on the body. Our study showed that acute stress reactivity was related with basal HPA axis activity, and for the first time showed that habituation to repeated psychological stressor was related with basal HPA axis activity. Considering the health implications of basal HPA axis activity reported by previous literature, particular in longitudinal studies, our finding can serve as an important step in bridging the gap of current literature about the health effects of acute stress reactivity and health outcomes of basal HPA axis activity, suggesting a model that maladaptive stress response patterns and HPA axis basal activity were related, and therefore leading to adverse health outcomes.

Our study investigated the relationship between cortisol and inflammatotry reactivity to acute stress and dynamics of basal HPA axis activity, and to the best of our knowledge, it is the first time that a direct relationship between habituation of repeated stress exposure and the basal HPA axis activity was reported. Therefore, it can be an important step of understanding 
the health effect of acute stress reactivity, both during initial response and upon repeated exposure.

\subsection{Limitations}

These findings have to be interpreted in light of some limitations. First, we used a crosssectional design and currently we do not have enough information about the directionality of these relationships to draw causal conclusions. We hypothesized that maladaptive response patterns, when repeatedly activated in daily life, change basal activity through prolonged exposure to cortisol and inflammatory products, but this specific directionality cannot be tested with our current design. Nevertheless, the identification of this relationship is important in terms of understanding the potential long-term effect of acute stress reactivity.

Second, day-to-day variation of basal cortisol activity based on short-term determinants such as the stress level of a particular day was documented in some studies (Adam et al., 2006; Wetherell et al., 2015b). But generally speaking, cortisol diurnal curve is a relative stable trait (Edwards et al., 2001; Elder et al., 2016; Pruessner et al., 1997a), and we tried to control for the effect of a particular day by asking the participants to take home samples on two consecutive days and using the average value. Further, our analysis of any effect of sampling day using repeated measures ANOVA yielded non-significant results, meaning that most likely, the effect of a particular day is not major concern in our data set.

Our finding that greater curvilinearity of daily cortisol curve, that is, the tendency to slow down both CAR linear increase slope and daily linear decline slope, was related with more adaptive acute stress reactivity is novel. The biological basis of curvilinearity could be in the negative feedback of the HPA axis, but it needs to be determined at which level negative feedback is compromised, and interactions need to be quantitatively studied. In future studies, we plan to study the HPA axis as a dynamic system and build differential equation groups to model the details of this biological system (Aschbacher et al., 2012). 


\subsection{Conclusions}

Taken together, our findings suggest that HPA axis and inflammatory response patterns to acute stress and basal HPA axis activity are related. Stronger reactivity to acute stressors and better habituation are related with patterns of basal cortisol activity that have been linked to positive health outcomes. This is an important step in understanding the long-term health implications of acute stress responsiveness. Future studies should employ longitudinal designs that can better identify the direction of these relationships. Further, a mechanistic study of the HPA axis that takes the interaction of different levels of this system into consideration should be performed to study the details of the dynamic system.

\section{Acknowledgements}

This research was supported by the American Federation of Aging Research (NR), and by training grants from the National Institute of Health (T32-084907 DG). MVT acknowledges funding from the Swiss National Science Foundation (SNF). We also want to thank research assistants in the Laboratory for Biological Health Psychology at Brandeis University for their help with data collection. 


\section{References}

Adam, E.K., 2006. Transactions among adolescent trait and state emotion and diurnal and momentary cortisol activity in naturalistic settings. Psychoneuroendocrinology 31, 664-679.

Adam, E.K., Hawkley, L.C., Kudielka, B.M., Cacioppo, J.T., 2006. Day-to-day dynamics of experience--cortisol associations in a population-based sample of older adults. Proceedings of the National Academy of Sciences of the United States of America 103, 17058-17063.

Adam, E.K., Kumari, M., 2009. Assessing salivary cortisol in large-scale, epidemiological research. Psychoneuroendocrinology 34, 1423-1436.

Aschbacher, K., Adam, E.K., Crofford, L.J., Kemeny, M.E., Demitrack, M.A., Ben-Zvi, A., 2012. Linking disease symptoms and subtypes with personalized systems-based phenotypes: a proof of concept study. Brain, behavior, and immunity 26, 1047-1056.

Besedovsky, H.O., del Rey, A., Klusman, I., Furukawa, H., Monge Arditi, G., Kabiersch, A., 1991. Cytokines as modulators of the hypothalamus-pituitary-adrenal axis. The Journal of steroid biochemistry and molecular biology 40, 613-618.

Breines, J.G., Thoma, M.V., Gianferante, D., Hanlin, L., Chen, X., Rohleder, N., 2014. Selfcompassion as a predictor of interleukin- 6 response to acute psychosocial stress. Brain, behavior, and immunity 37, 109-114.

Brydon, L., Steptoe, A., 2005. Stress-induced increases in interleukin-6 and fibrinogen predict ambulatory blood pressure at 3-year follow-up. Journal of hypertension 23, 1001-1007.

Bryk, A.S., Raudenbush, S.W., 1992. Hierarchical linear models: applications and data analysis methods. Sage Publications.

Burke, H.M., Davis, M.C., Otte, C., Mohr, D.C., 2005. Depression and cortisol responses to psychological stress: a meta-analysis. Psychoneuroendocrinology 30, 846-856.

Buske-Kirschbaum, A., Geiben, A., Hollig, H., Morschhauser, E., Hellhammer, D., 2002. Altered responsiveness of the hypothalamus-pituitary-adrenal axis and the sympathetic adrenomedullary system to stress in patients with atopic dermatitis. The Journal of clinical endocrinology and metabolism 87, 4245-4251.

Cermakian, N., Westfall, S., Kiessling, S., 2014. Circadian clocks and inflammation: reciprocal regulation and shared mediators. Archivum immunologiae et therapiae experimentalis 62, 303318.

Champaneri, S., Xu, X., Carnethon, M.R., Bertoni, A.G., Seeman, T., DeSantis, A.S., Diez Roux, A., Shrager, S., Golden, S.H., 2013. Diurnal salivary cortisol is associated with body mass index and waist circumference: the Multiethnic Study of Atherosclerosis. Obesity 21, E5663.

Chida, Y., Steptoe, A., 2009. Cortisol awakening response and psychosocial factors: A systematic review and meta-analysis. Biol Psychol 80, 265-278. 
Chrousos, G.P., Gold, P.W., 1992. The concepts of stress and stress system disorders. Overview of physical and behavioral homeostasis. JAMA : the journal of the American Medical Association 267, 1244-1252.

Cohen, S., Hamrick, N., 2003. Stable individual differences in physiological response to stressors: implications for stress-elicited changes in immune related health. Brain, behavior, and immunity $17,407-414$.

Cohen, S., Janicki-Deverts, D., Miller, G.E., 2007. Psychological stress and disease. JAMA : the journal of the American Medical Association 298, 1685-1687.

Dantzer, R., O'Connor, J.C., Freund, G.G., Johnson, R.W., Kelley, K.W., 2008. From inflammation to sickness and depression: when the immune system subjugates the brain. Nature reviews. Neuroscience 9, 46-56.

Dickerson, S.S., Kemeny, M.E., 2004. Acute stressors and cortisol responses: a theoretical integration and synthesis of laboratory research. Psychological bulletin 130, 355-391.

Dmitrieva, N.O., Almeida, D.M., Dmitrieva, J., Loken, E., Pieper, C.F., 2013. A day-centered approach to modeling cortisol: Diurnal cortisol profiles and their associations among U.S. adults. Psychoneuroendocrinology.

Doane, L.D., Adam, E.K., 2010. Loneliness and cortisol: momentary, day-to-day, and trait associations. Psychoneuroendocrinology 35, 430-441.

Duan, H., Yuan, Y., Zhang, L., Qin, S., Zhang, K., Buchanan, T.W., Wu, J., 2013. Chronic stress exposure decreases the cortisol awakening response in healthy young men. Stress 16, 630637.

Dunn, A.J., 2000. Cytokine activation of the HPA axis. Annals of the New York Academy of Sciences 917, 608-617.

Edwards, S., Clow, A., Evans, P., Hucklebridge, F., 2001. Exploration of the awakening cortisol response in relation to diurnal cortisol secretory activity. Life sciences 68, 2093-2103.

Elder, G.J., Ellis, J.G., Barclay, N.L., Wetherell, M.A., 2016. Assessing the daily stability of the cortisol awakening response in a controlled environment. BMC Psychology 4, 1-10.

Gianferante, D., Thoma, M.V., Hanlin, L., Chen, X., Breines, J.G., Zoccola, P.M., Rohleder, N., 2014. Post-stress rumination predicts HPA axis responses to repeated acute stress.

Psychoneuroendocrinology 49, 244-252.

Hackett, R.A., Kivimäki, M., Kumari, M., Steptoe, A., 2016. Diurnal Cortisol Patterns, Future Diabetes, and Impaired Glucose Metabolism in the Whitehall II Cohort Study. J. Clin. Endocrinol. Metab. 101, 619-625 617p.

Hamer, M., Steptoe, A., 2012. Cortisol responses to mental stress and incident hypertension in healthy men and women. The Journal of clinical endocrinology and metabolism 97, E29-34.

Heaney, J.L., Phillips, A.C., Carroll, D., 2012. Aging, health behaviors, and the diurnal rhythm and awakening response of salivary cortisol. Experimental aging research 38, 295-314. 
Jarcho, M.R., Slavich, G.M., Tylova-Stein, H., Wolkowitz, O.M., Burke, H.M., 2013.

Dysregulated diurnal cortisol pattern is associated with glucocorticoid resistance in women with major depressive disorder. Biol Psychol 93, 150-158.

Juster, R.P., McEwen, B.S., Lupien, S.J., 2010. Allostatic load biomarkers of chronic stress and impact on health and cognition. Neurosci Biobehav Rev 35, 2-16.

Kemeny, M.E., 2003. The psychobiology of stress. Curr. Dir. Psychol. 12, 124-129.

Kidd, T., Carvalho, L.A., Steptoe, A., 2014. The relationship between cortisol responses to laboratory stress and cortisol profiles in daily life. Biol Psychol 99, 34-40.

Kirschbaum, C., Hellhammer, D.H., 1989. Salivary cortisol in psychobiological research - an overview. Neuropsychobiology 22, 150-169.

Kirschbaum, C., Pirke, K.M., Hellhammer, D.H., 1993. The 'Trier Social Stress Test'--a tool for investigating psychobiological stress responses in a laboratory setting. Neuropsychobiology 28 , 76-81.

Kirschbaum, C., Prussner, J.C., Stone, A.A., Federenko, I., Gaab, J., Lintz, D., Schommer, N., Hellhammer, D.H., 1995. Persistent high cortisol responses to repeated psychological stress in a subpopulation of healthy men. Psychosomatic medicine 57, 468-474.

Knight, J.M., Avery, E.F., Janssen, I., Powell, L.H., 2010. Cortisol and depressive symptoms in a population-based cohort of midlife women. Psychosomatic medicine 72, 855-861.

Kudielka, B.M., Hellhammer, D.H., Kirschbaum, C., 2007. Ten years of research with the trier social stress test--revisited, in: Harmon-Jones, E., Winkielman, P., Harmon-Jones, E., Winkielman, P. (Eds.), Social neuroscience: Integrating biological and psychological explanations of social behavior. Guilford Press, New York, NY, US, pp. 56-83.

Kudielka, B.M., R., v.K., Preckel, D., Zgraggen, L., Mischler, K., Fischer, J.E., 2006. Exhaustion is associated with reduced habituation of free cortisol responses to repeated acute psychosocial stress. Biol Psychol 72, 147-153.

Kumari, M., Chandola, T., Brunner, E., Kivimaki, M., 2010. A nonlinear relationship of generalized and central obesity with diurnal cortisol secretion in the Whitehall II study. The Journal of clinical endocrinology and metabolism 95, 4415-4423.

Kumari, M., Shipley, M., Stafford, M., Kivimaki, M., 2011. Association of diurnal patterns in salivary cortisol with all-cause and cardiovascular mortality: findings from the Whitehall II study. The Journal of clinical endocrinology and metabolism 96, 1478-1485.

Kunz-Ebrecht, S.R., Kirschbaum, C., Marmot, M., Steptoe, A., 2004. Differences in cortisol awakening response on work days and weekends in women and men from the Whitehall II cohort. Psychoneuroendocrinology 29, 516-528.

Labonte, B., Azoulay, N., Yerko, V., Turecki, G., Brunet, A., 2014. Epigenetic modulation of glucocorticoid receptors in posttraumatic stress disorder. Translational psychiatry 4, e368. 
Laufer, D., Ansermet, F., von der Weid, N., Beck Popovic, M., Torrisi, R., Pierrehumbert, B., 2012. Endocrine response and perceived stress test during an experimental challenge task in adult survivors of a childhood cancer. Pediatric blood \& cancer 59, 138-143.

Lightman, S.L., Conway-Campbell, B.L., 2010. The crucial role of pulsatile activity of the HPA axis for continuous dynamic equilibration. Nature reviews. Neuroscience 11, 710-718.

Lucertini, F., Ponzio, E., Di Palma, M., Galati, C., Federici, A., Barbadoro, P., D'Errico, M.M., Prospero, E., Ambrogini, P., Cuppini, R., Lattanzi, D., Minelli, A., 2015. High Cardiorespiratory Fitness Is Negatively Associated with Daily Cortisol Output in Healthy Aging Men. PloS one 10, e0141970.

McEwen, B.S., 1998. Protective and damaging effects of stress mediators. The New England journal of medicine $338,171-179$.

McEwen, B.S., Stellar, E., 1993. Stress and the individual. Mechanisms leading to disease. Archives of internal medicine 153, 2093-2101.

Miller, G.E., Chen, E., Zhou, E.S., 2007. If it goes up, must it come down? Chronic stress and the hypothalamic-pituitary-adrenocortical axis in humans. Psychological bulletin 133, 25-45.

Nader, N., Chrousos, G.P., Kino, T., 2010. Interactions of the circadian CLOCK system and the HPA axis. Trends in endocrinology and metabolism: TEM 21, 277-286.

Pruessner, J.C., Wolf, O.T., Hellhammer, D.H., Buske-Kirschbaum, A., von Auer, K., Jobst, S., Kaspers, F., Kirschbaum, C., 1997a. Free cortisol levels after awakening: a reliable biological marker for the assessment of adrenocortical activity. Life sciences 61, 2539-2549.

Pruessner, J.C., Wolf, O.T., Hellhammer, D.H., BuskeKirschbaum, A., vonAuer, K., Jobst, S., Kaspers, F., Kirschbaum, C., 1997b. Free cortisol levels after awakening: A reliable biological marker for the assessment of adrenocortical activity. Life sciences 61, 2539-2549.

Quirin, M., Pruessner, J.C., Kuhl, J., 2008. HPA system regulation and adult attachment anxiety: Individual differences in reactive and awakening cortisol. Psychoneuroendocrinology 33, 581590.

Ridker, P.M., Rifai, N., Stampfer, M.J., Hennekens, C.H., 2000. Plasma concentration of interleukin- 6 and the risk of future myocardial infarction among apparently healthy men. Circulation 101, 1767-1772.

Rohleder, N., 2012. Acute and chronic stress induced changes in sensitivity of peripheral inflammatory pathways to the signals of multiple stress systems --2011 Curt Richter Award Winner. Psychoneuroendocrinology 37, 307-316.

Rohleder, N., 2014. Stimulation of systemic low-grade inflammation by psychosocial stress. Psychosomatic medicine 76, 181-189.

Sapolsky, R.M., 1999. Glucocorticoids, stress, and their adverse neurological effects: relevance to aging. Exp. Gerontol. 34, 721-732. 
Sapolsky, R.M., Romero, L.M., Munck, A.U., 2000. How do glucocorticoids influence stress responses? Integrating permissive, suppressive, stimulatory, and preparative actions. Endocr Rev 21, 55-89.

Sarabdjitsingh, R.A., Isenia, S., Polman, A., Mijalkovic, J., Lachize, S., Datson, N., de Kloet, E.R., Meijer, O.C., 2010. Disrupted corticosterone pulsatile patterns attenuate responsiveness to glucocorticoid signaling in rat brain. Endocrinology 151, 1177-1186.

Scheiermann, C., Kunisaki, Y., Frenette, P.S., 2013. Circadian control of the immune system. Nature reviews. Immunology 13, 190-198.

Schmidt-Reinwald, A., Pruessner, J.C., Hellhammer, D.H., Federenko, I., Rohleder, N., Schurmeyer, T.H., Kirschbaum, C., 1999. The cortisol response to awakening in relation to different challenge tests and a 12-hour cortisol rhythm. Life sciences 64, 1653-1660.

Schommer, N.C., Hellhammer, D.H., Kirschbaum, C., 2003. Dissociation between reactivity of the hypothalamus-pituitary-adrenal axis and the sympathetic-adrenal-medullary system to repeated psychosocial stress. Psychosomatic medicine 65, 450-460.

Schrepf, A., Thaker, P.H., Goodheart, M.J., Bender, D., Slavich, G.M., Dahmoush, L., Penedo, F., DeGeest, K., Mendez, L., Lubaroff, D.M., Cole, S.W., Sood, A.K., Lutgendorf, S.K., 2015. Diurnal cortisol and survival in epithelial ovarian cancer. Psychoneuroendocrinology 53, 256267.

Sephton, S.E., Lush, E., Dedert, E.A., Floyd, A.R., Rebholz, W.N., Dhabhar, F.S., Spiegel, D., Salmon, P., 2013. Diurnal cortisol rhythm as a predictor of lung cancer survival. Brain, behavior, and immunity 30 Suppl, S163-170.

Sephton, S.E., Sapolsky, R.M., Kraemer, H.C., Spiegel, D., 2000. Diurnal cortisol rhythm as a predictor of breast cancer survival. Journal of the National Cancer Institute 92, 994-1000.

Siddiqui, A., Madhu, S.V., Sharma, S.B., Desai, N.G., 2015. Endocrine stress responses and risk of type 2 diabetes mellitus. Stress 18, 498-506.

Slatcher, R.B., Chi, P., Li, X., Zhao, J., Zhao, G., Ren, X., Zhu, J., Stanton, B., 2015. Associations between coping and diurnal cortisol among children affected by parental HIVIAIDS. Health psychology : official journal of the Division of Health Psychology, American Psychological Association.

Stafford, M., Gardner, M., Kumari, M., Kuh, D., Ben-Shlomo, Y., 2013. Social isolation and diurnal cortisol patterns in an ageing cohort. Psychoneuroendocrinology 38, 2737-2745.

Stawski, R.S., Almeida, D.M., Lachman, M.E., Tun, P.A., Rosnick, C.B., Seeman, T., 2011. Associations between cognitive function and naturally occurring daily cortisol during middle adulthood: timing is everything. The journals of gerontology. Series B, Psychological sciences and social sciences 66 Suppl 1, i71-81.

Sternberg, E.M., Chrousos, G.P., Wilder, R.L., Gold, P.W., 1992. The stress response and the regulation of inflammatory disease. Annals of internal medicine 117, 854-866. 
Tomiyama, A.J., O'Donovan, A., Lin, J., Puterman, E., Lazaro, A., Chan, J., Dhabhar, F.S., Wolkowitz, O., Kirschbaum, C., Blackburn, E., Epel, E., 2012. Does cellular aging relate to patterns of allostasis? An examination of basal and stress reactive HPA axis activity and telomere length. Physiology \& behavior 106, 40-45.

Turnbull, A.V., Rivier, C., 1995. Regulation of the HPA axis by cytokines. Brain, behavior, and immunity $9,253-275$.

Veen, G., van Vliet, I.M., DeRijk, R.H., Giltay, E.J., van Pelt, J., Zitman, F.G., 2011. Basal cortisol levels in relation to dimensions and DSM-IV categories of depression and anxiety. Psychiatry research 185, 121-128.

von Kanel, R., Dimsdale, J.E., Mills, P.J., Ancoli-Israel, S., Patterson, T.L., Mausbach, B.T., Grant, I., 2006a. Effect of Alzheimer caregiving stress and age on frailty markers interleukin-6, C-reactive protein, and D-dimer. The journals of gerontology. Series A, Biological sciences and medical sciences 61, 963-969.

von Kanel, R., Kudielka, B.M., Preckel, D., Hanebuth, D., Fischer, J.E., 2006b. Delayed response and lack of habituation in plasma interleukin- 6 to acute mental stress in men. Brain, behavior, and immunity $20,40-48$.

Vreeburg, S.A., Hoogendijk, W.J., DeRijk, R.H., van Dyck, R., Smit, J.H., Zitman, F.G., Penninx, B.W., 2013. Salivary cortisol levels and the 2-year course of depressive and anxiety disorders. Psychoneuroendocrinology 38, 1494-1502.

Vreeburg, S.A., Kruijtzer, B.P., van Pelt, J., van Dyck, R., DeRijk, R.H., Hoogendijk, W.J., Smit, J.H., Zitman, F.G., Penninx, B.W., 2009. Associations between sociodemographic, sampling and health factors and various salivary cortisol indicators in a large sample without psychopathology. Psychoneuroendocrinology 34, 1109-1120.

Weitzman, E.D., Fukushima, D., Nogeire, C., Roffwarg, H., Gallagher, T.F., Hellman, L., 1971. Twenty-four hour pattern of the episodic secretion of cortisol in normal subjects. The Journal of clinical endocrinology and metabolism 33, 14-22.

Wellen, K.E., Hotamisligil, G.S., 2005. Inflammation, stress, and diabetes. The Journal of clinical investigation 115, 1111-1119.

Wetherell, M., Montgomery, C., Smith, M., 2015a. The effects of anticipated challenge on indices of diurnal cortisol secretion in recreational users of ecstasy. Psychoneuroendocrinology $61,58$.

Wetherell, M.A., Lovell, B., Smith, M.A., 2015b. The effects of an anticipated challenge on diurnal cortisol secretion. Stress 18.

Wust, S., Wolf, J., Hellhammer, D.H., Federenko, I., Schommer, N., Kirschbaum, C., 2000. The cortisol awakening response - normal values and confounds. Noise \& health 2, 79-88. 


\section{Figure Legends}

Figure 1: $(A)$ and $(B)$ show HPA axis and inflammatory responses to repeated stress. Both HPA axis $(A)$ and the inflammatory system $(B)$ were activated to acute stress. During repeated exposure, HPA axis showed habituation while IL-6 showed sensitization. (C) shows the diurnal curve of HPA axis, with a rapid increase in the first hour upon awakening and a slow decline throughout rest of the day.

Figure 2: Factors that were related with CAR. (A) Young participants generally had higher levels of cortisol than older participants, and females had steeper linear increase of CAR. (B) Participants with higher cortisol responses to initial stress had a steeper linear increase and greater curvilinearity. (C) Participants with stronger cortisol habituation had a steeper linear increase, and greater curvilinearity $\left(\mathrm{Q} 1=1^{\text {st }}\right.$ quartile, $Q 3=3^{\text {rd }}$ quartile).

Figure 3: (A) Participants with higher cortisol responses to initial stress had steeper linear decreases of diurnal cortisol. (B) Participants with stronger cortisol habituation had steeper linear decreases and greater curvilinearity of diurnal cortisol (Q1=1st quartile, $Q 3=3 r$ quartile).

Figure 4: (A) Participants with higher IL-6 response during first stress exposure had steeper daily cortisol decrease and greater curvilinearity. (B) Participants with stronger IL-6 habituation had steeper daily cortisol decrease and greater curvilinearity (Q1= 1st quartile, Q3= 3rd quartile). 
10. Table 1: Demographic information

\begin{tabular}{|c|c|c|}
\hline & Young group & Older group \\
\hline Age & $21.30 \pm 4.43$ years & $54.47 \pm 4.42$ years \\
\hline Gender & 18 male, 15 female & 6 male, 9 female \\
\hline \multirow[t]{5}{*}{ Race } & African American: $6.3 \%$; & African American:6.3\% \\
\hline & Asian: $37.5 \%$ & Asian: $0 \%$ \\
\hline & Caucasian: $50 \%$ & Caucasian: $75 \%$ \\
\hline & Other: $6.5 \%$ & Other or more than one race: $12.6 \%$ \\
\hline & Do not wish to reply: $0 \%$ & Do not wish to reply: $6.3 \%$ \\
\hline BMI & $24.27 \pm 3.02 \mathrm{~kg} / \mathrm{m}^{2}$ & $25.08 \pm 3.33 \mathrm{~kg} / \mathrm{m}^{2}$ \\
\hline \multirow[t]{3}{*}{ Education } & $75 \%$ undergraduate student & $12.5 \%$ did not finish college \\
\hline & $6.3 \%$ with Bachelor's degree & $43.8 \%$ with Bachelor's degree \\
\hline & $18.8 \%$ with higher degree & $43.9 \%$ with higher degree \\
\hline
\end{tabular}



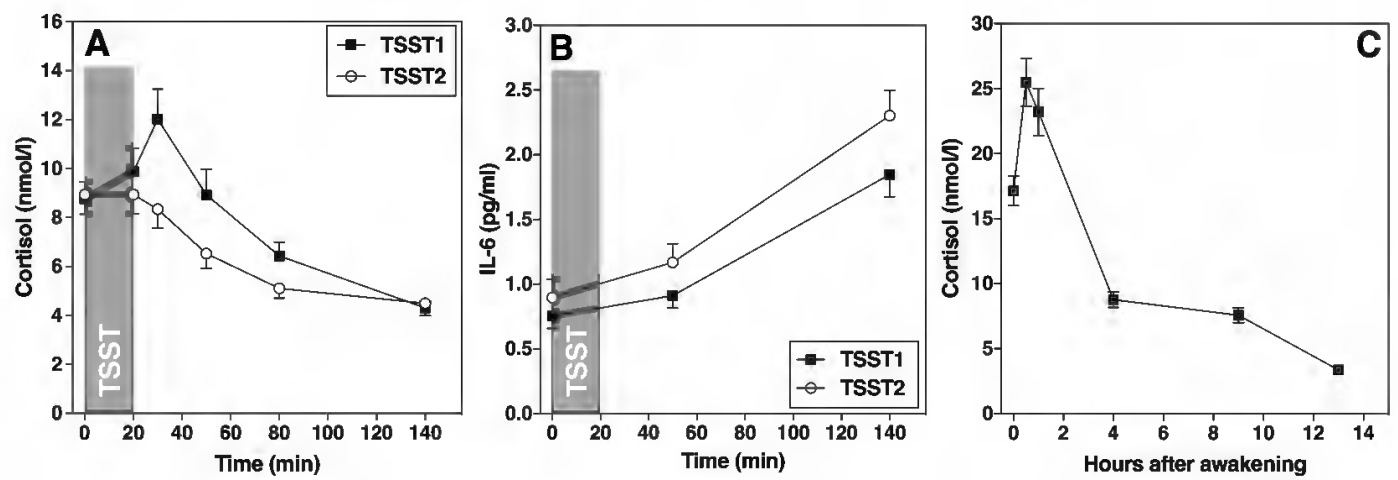

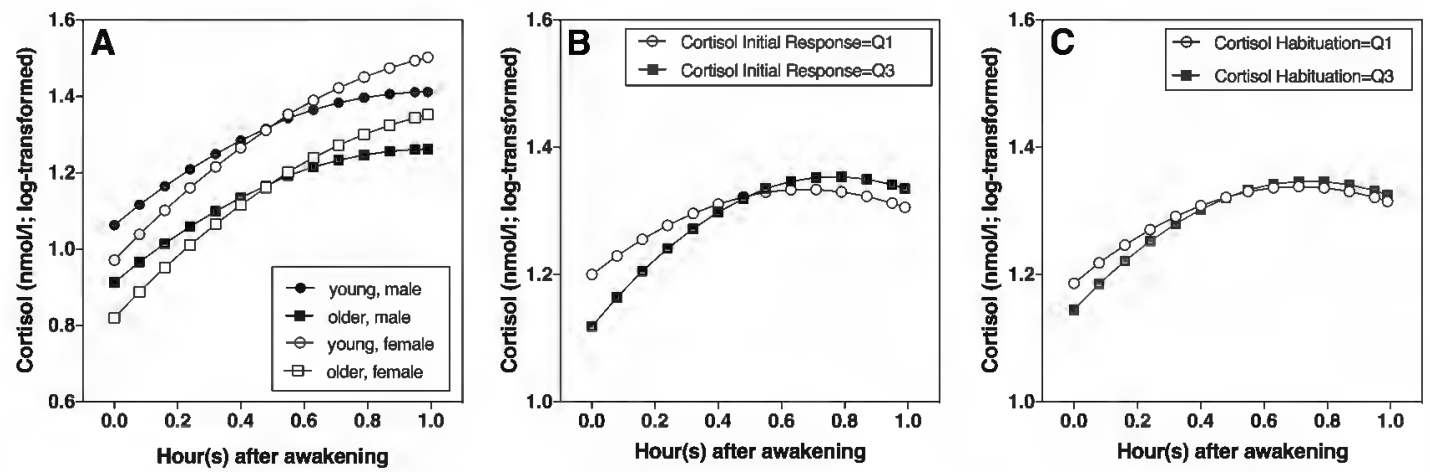

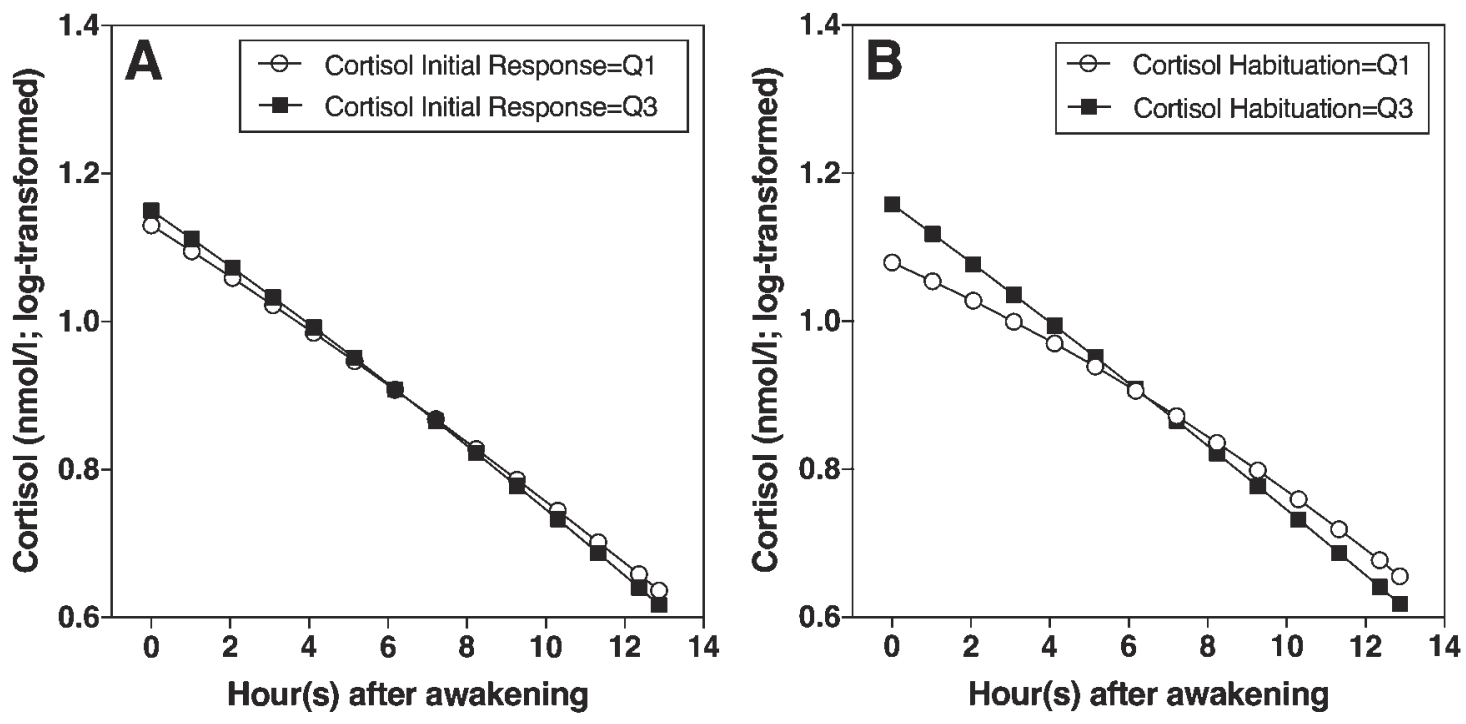

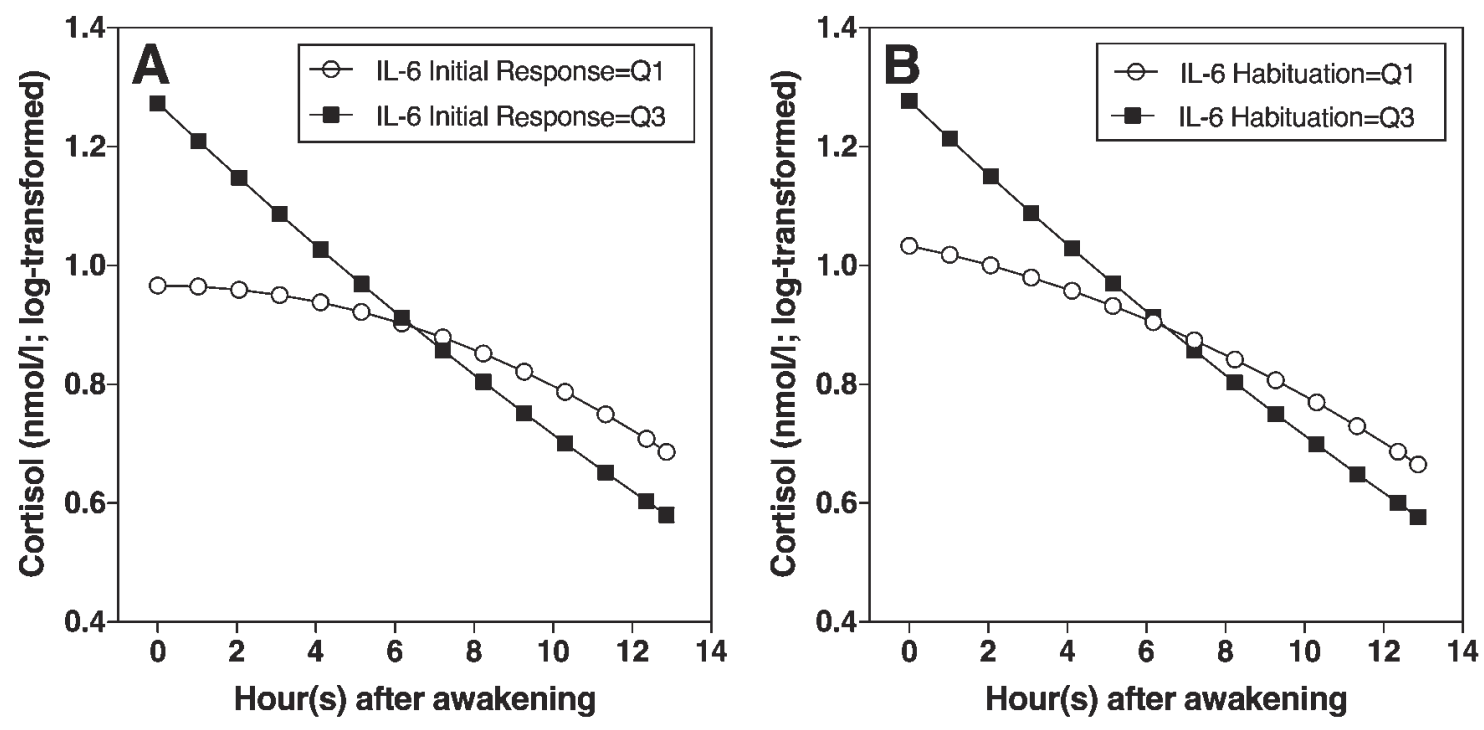